\title{
New method for the study of Amaryllidaceae alkaloid biosynthesis using biotransformation of deuterium-labeled precursor in tissue cultures
}

\author{
Anna El Tahchy1', Michel Boisbrun'1, Agata Ptak², François Dupire², Françoise Chrétien1, \\ Max Henry' ${ }^{1}$, Yves Chapleur ${ }^{1}$ and Dominique Laurain-Mattar ${ }^{1 \times}$
}

${ }^{1}$ Groupe S.U.C.R.E.S., UMR 7565 CNRS-Nancy-Université, Nancy-Vandoeuvre, France; 2Department of Plant Breeding and Seed Science,

Agricultural University, Kraków, Poland; ${ }^{3 S}$ ervice Commun de Spectrométrie de Masse, UHP-Institut Jean Barriol, Nancy-Vandoeuvre, France

\begin{abstract}
Biotransformation of deuterated-4'-O-methylnorbelladine into alkaloids galanthamine and lycorine in tissue cultures of Leucojum aestivum was demonstrated using HPLC coupled to mass spectrometry. GC-MS screening was also carried to investigate other native and deuterated alkaloids. A total of six labeled alkaloids were identified indicating that $4^{\prime}$-O-methyl- $\mathrm{d}_{3}$-norbelladine is incorporated into three different groups of Amaryllidaceae alkaloids that are biosynthesized by three modes of intramolecular oxidative phenol coupling.
\end{abstract}

Keywords: alkaloids, Leucojum aestivum, Amaryllidaceae, deuterated precursor, biotransformation

Received: 15 October, 2009; revised: 05 December, 2009; accepted: 15 December, 2009; available on-line: 11 January, 2010

\section{INTRODUCTION}

Amaryllidaceae alkaloids have important pharmacological properties such as acetylcholinesterase inhibitory activity, cytotoxicity and antitumoral activity (Bastida et al., 2006). Galanthamine, an isoquinoline alkaloid, is obtained on a commercial scale for its pharmacological interest from Narcissus spp. and Leucojum aestivum as well as synthetically (Guillou et al., 2001; Marco-Contelles et al., 2006). Lycorine, a pyrrolophenanthridine alkaloid, displays a strong antiviral effect against poliovirus, measles and Herpes simplex type 1 viruses, as well as high antiretroviral (Szlávik et al., 2004) and strong antimitotic activities (Kukhanova et al., 1983).

Several studies on the biosynthesis of Amaryllidaceae alkaloids belonging to different ring type subgroups have been reported (Barton \& Cohen, 1957; Barton et al., 1963; Bernfeld, 1963; Eichhorn et al., 1998; Herbert, 2001; Kornienko \& Evidente, 2008). All Amaryllidaceae alkaloids can be regarded as derivatives of the common precursor 4'-O-methylnorbelladine via intramolecular oxidative phenol-coupling (Barton \& Cohen, 1957; Barton et al., 1963). There are three different groups of Amaryllidaceae alkaloids that are biosynthesized by three modes of intramolecular oxidative phenol coupling of the common precursor (Fig. 1) (Herbert, 2001; Kornienko \& Evidente, 2008). The para-ortho' oxidative coupling leads to a dienone which spontaneously cyclizes to demethylnarwedine which upon stereoselective reduction leads to demethylgalanthamine. Subsequent N-methylation of this compound gives galanthamine. Narwedine exists in equilibrium with galanthamine, a reaction catalyzed by a hypothetically reversible oxidoreductase (Eichhorn et al., 1998). para-para' coupling gives another dienone which, depending on the nitrogen nucleophilic addition site, gives rise to marithidine or crinine derivatives. Finally, ortho-para' coupling yields lycorine.

Nevertheless, the biosynthetic pathway of Amaryllidaceae alkaloids, particularly in Leucojum aestivum, has not been totally elucidated yet. Eichhorn et al. (1998) have established a revised scheme for the biosynthesis of galanthamine (Fig. 1, para-ortho' oxidative coupling of 4'-O-methylnorbelladine).

The investigations on the biosynthesis of Amaryllida-

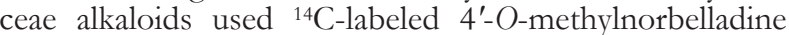
injected into organs of daffodil plants or ${ }^{13} \mathrm{C}$ or ${ }^{3} \mathrm{H}_{3} \mathrm{C}-\mathrm{la}-$ beled 4'-O-methylnorbelladine applied to organs of field grown L. aestivum plants (Barton et al., 1963; Eichhorn et al., 1998).

Here we report the development of a rapid and convenient method for the study of the biosynthetic pathway of Amaryllidaceae alkaloids using a method based on deuterium labeled precursor fed to in vitro cultures of $L$. aestivum. This paper reports for the first time the biotransformation of the common precursor 4'-O-meth$y \mathrm{l}-\mathrm{d}_{3}-$ norbelladine in shoot cultures of $L$. aestivum. Mass spectrometry was used for the identification of the labeled alkaloids. HPLCMS was used for the analysis of deuterated galanthamine and lycorine in tissue cultures and in the liquid medium. GC-MS analyses are also reported for the screening and identification of other deuterated alkaloids.

\section{MATERIALS AND METHODS}

Plant material. Leaves isolated from Leucojum aestivum L. bulbs (from French local markets) chilled for 12 weeks at $5{ }^{\circ} \mathrm{C}$ were surface-sterilized in $70 \%$ ethanol (1 min), then shaken for $15 \mathrm{~min}$ in $15 \%$ Domestos (with sodium hypochlorite and sodium hydroxide content below $5 \%$; Unilever, Hungary) and rinsed three times with sterile water. Sterilized leaves were cut into thin slices (about 2 to $3 \mathrm{~mm}$ in length) and plated on culture medium.

- e-mail: dominique.laurain-mattar@pharma.uhp-nancy.fr

Abbreviations: AnhydroLYC, anhydrolycorine; BAP, 6-benzylaminopurine; $\mathrm{CRI}$, crinine; DemethylGAL, demethylgalanthamine; DemethylMAR, demethylmarithidine; D3MN, 4'-O-methyl-d $\mathrm{d}_{3}$-norbelladine; GAL, galanthamine; NAA, naphthalene-1-acetic acid; $N$ FGAL, N-formylnorgalanthamine; LYC, lycorine; NAR, narwedine; $\mathrm{N}$-demethyINAR, $\mathrm{N}$-demethylnarwedine; TRIS, trisphaeridine. 
Shoot cultures. Initial explants were cultivated on Murashige and Skoog (1962) medium (Murashige \& Skoog, 1962) supplemented with auxin $\alpha$-naphthalene acetic acid (NAA) $(10 \mu \mathrm{M})$ and cytokinin benzylaminopurine (BAP) (5 $\mu \mathrm{M})$ (control). The medium was supplemented with $3 \%$ sucrose, adjusted to $\mathrm{pH} 5.5$ before autoclaving and gelled with $0.8 \%$ purified agar (Difco). The cultures were maintained at $25 \pm 2{ }^{\circ} \mathrm{C}$ in the darkness and subcultured every 4 weeks. Twelve month old shoot cultures $(2 \mathrm{~g})$ were submerged in liquid-shake medium $(20 \mathrm{~mL})$ containing labeled precursor 4'-O-methyl$\mathrm{d}_{3}$-norbelladine at various concentrations $(0.05,0.10$ and $0.20 \mathrm{~g} / \mathrm{L}$ ) and incubated at $25 \pm 2{ }^{\circ} \mathrm{C}$ in the darkness for various periods of time (15, 30 and 40 days). A stock solution for feeding experiments was prepared from 50 $\mathrm{mg}$ of $4^{\prime}-\mathrm{O}$-methyl- $\mathrm{d}_{3}$-norbelladine dissolved in $10 \mathrm{~mL}$ of $\mathrm{H}_{2} \mathrm{O}$. Solutions at appropriate concentrations of the labeled precursor were filter-sterilized directly into the flasks.

Chemicals and reagents. Triethylammonium acetate buffer, galanthamine, lycorine, 6-benzylaminopurine, inositol, agar-agar, magnesium sulphate heptahydrate (98\%), manganese sulphate, potassium nitrate, and ethylenediaminetetraacetic acid iron (III) sodium salt were all from Sigma-Aldrich Chemie GmbH (Stennheim, Germany). Ammonium bicarbonate, iron sulphate, zinc sulphate, copper(II)sulphate pentahydrate $(99 \%)$, thiamine hydrochloride, $\alpha$-naphthalene acetic acid, absolute ethanol, and methanol (Hipersolv Chromanorum for HPLC Isocratic Grade) were purchased from Prolabo VWR international bvba/sprl. Pyridoxol hydrochloride, nicotinic acid, potassium dihydrogenophosphate and calcium chloride dehydrate were obtained from Merck AG (Darmstadt, Germany). Acetonitrile was acquired from Carlo Erba Reagenti.

Synthesis of the labeled precursor. General experimental procedures: solvents were purified and dried according to recommended procedures. Melting points were measured on a Reichert Kofler apparatus and are uncorrected. Infra-red spectra were recorded on a Perkin-Elmer Spectrum 1000 FT-IR spectrometer. ${ }^{1} \mathrm{H}$ and ${ }^{13} \mathrm{C}$ NMR spectra were recorded on an AC 250 Bruker spectrometer (250 Mhz and $62.9 \mathrm{MHz}$, respectively). MS analysis of 3-hydroxy-4-methoxy- $\mathrm{d}_{3}$-benzaldehyde was

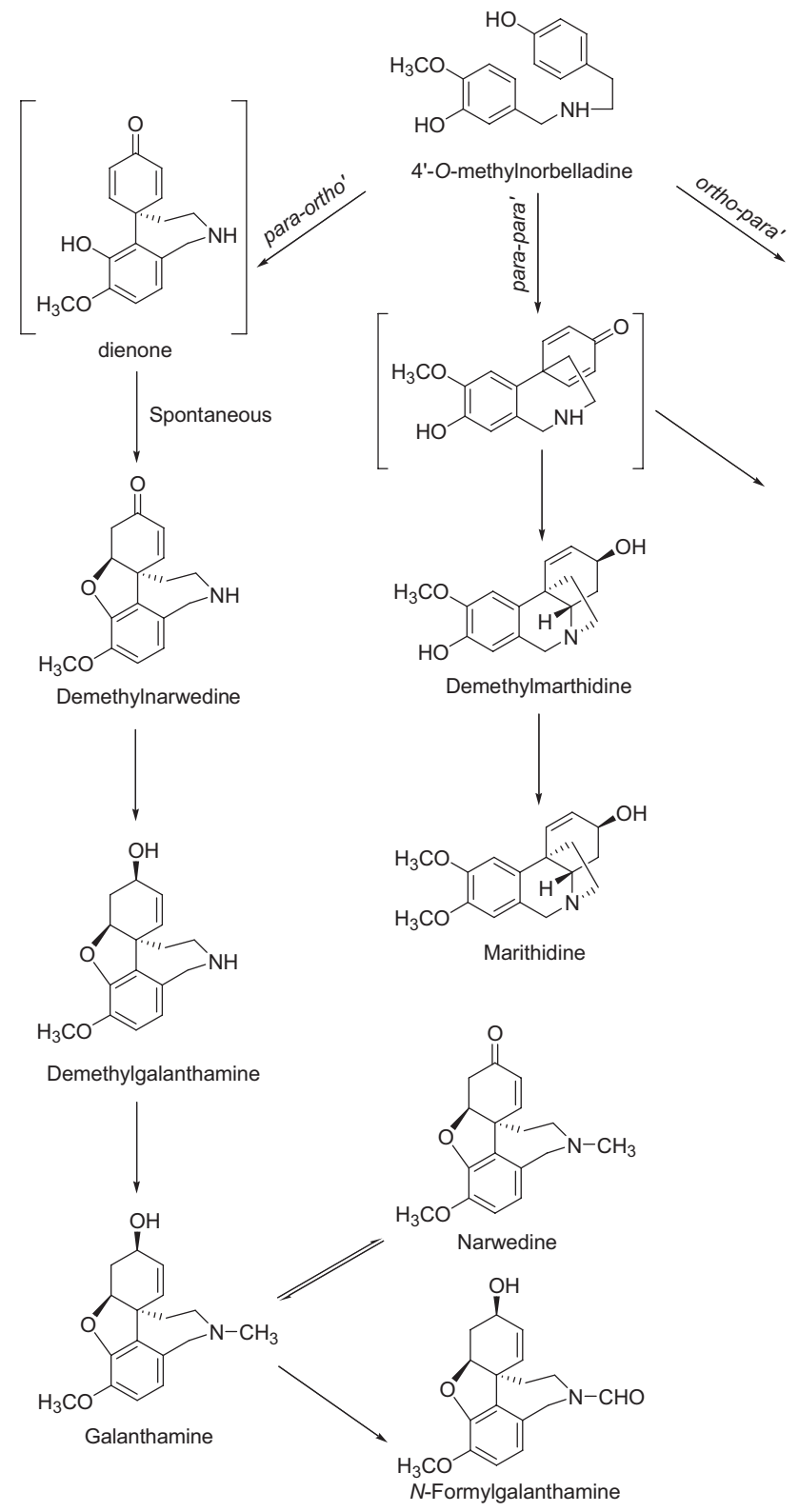<smiles></smiles><smiles>O=C1C=CC2(CC1)CN1Cc3cc2c(cc3OCO)C1</smiles>

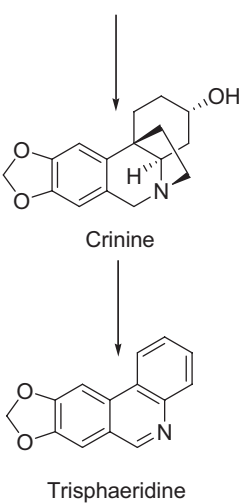

Figure 1. Postulated pathway for Amaryllidaceae alkaloid biosynthesis After (Eichhorn et al., 1998; Herbert, 2001; Kornienko \& Evidente, 2008). 
performed using QP2010 Shimadzu equipment operating in the CI mode. MS analysis of 4'-O-methyl- $\mathrm{d}_{3}$-norbelladine was performed using a micrOTOF ${ }_{\mathrm{Q}}^{\mathrm{TM}}$ (Bruker Daltonics) apparatus operating in the ES mode. Elemental analyses were performed on a Thermofinnigan FlashEA 1112 apparatus, at the Service Commun de Microanalyse (Nancy, France). Column chromatography was performed on silica gel SI $60(63-200 \mu \mathrm{m})$ (Merck).

3-Hydroxy-4-methoxy-d $\mathrm{d}_{3}$-benzaldehyde: to a suspension of finely powdered potassium carbonate (1.901 g, $13.76 \mathrm{mmol})$ in acetone $(70 \mathrm{~mL})$ was added 3,4-dihydroxybenzaldehyde $(1.900 \mathrm{~g}, 13.76 \mathrm{mmol})$ and methyl-d $\mathrm{d}_{3}-$ iodide $(856 \mu \mathrm{L}, 13.76 \mathrm{mmol})$. The mixture was refluxed under inert atmosphere for $8 \mathrm{~h}$, cooled and filtered. The solvent was evaporated and the residue was purified by column chromatography (eluent: ethyl acetate/hexane, $25: 75, \mathrm{v} / \mathrm{v})$ to give 3 -methoxy-d $\mathrm{d}_{3}-4$-hydroxy benzaldehyde $(0.300 \mathrm{~g}, 1.93 \mathrm{mmol})$, a mixture of the latter compound and the title compound $(0.612 \mathrm{~g}, 3.94 \mathrm{mmol})$, and the title compound $(0.628 \mathrm{~g}, 4.05 \mathrm{mmol})$. The mixture of the two isomers was re-purified the same way to give 3-methoxy- $\mathrm{d}_{3}$-4-hydroxy benzaldehyde $(0.107 \mathrm{~g}, 0.69$ $\mathrm{mmol})$ and the title compound $(0.427 \mathrm{~g}, 2.75 \mathrm{mmol})$. Total yield: $1.055 \mathrm{~g}$ (6.80 mmol, $49 \%$ yield $)$ of white crystals.

M.p. $111^{\circ} \mathrm{C}$ (lit.: $111^{\circ} \mathrm{C}$ ) (Markey et al., 1980) IR (KBr) $v_{\max } 3225,2865,1670,1605,1579,1508,1291,1251$, 1121, 1101, 991, 827, $632 \mathrm{~cm}^{-1} ;{ }^{1} \mathrm{H}$ NMR $\left(\mathrm{CDCl}_{3}\right): \delta$ 5.79 (s, $1 \mathrm{H}, \mathrm{OH}), 6.98\left(\mathrm{~d}, J=8.8 \mathrm{~Hz}, 1 \mathrm{H}, \mathrm{H}_{\text {arom }}\right), 7.44$ $\left(\mathrm{m}, 2 \mathrm{H}, \mathrm{H}_{\text {arom }}\right), 9.86(\mathrm{~s}, 1 \mathrm{H}, \mathrm{CHO}) ;{ }^{13} \mathrm{C} \mathrm{NMR}\left(\mathrm{CDCl}_{3}\right)$ : $\delta$ 110.4, 114.3, 124.8, 130.9, 146.3, 152.0, 191.3; CI-MS (pos. mode): $m / z 156[\mathrm{M}+\mathrm{H}]^{+}(100), 311[2 \mathrm{M}+\mathrm{H}]^{+}$ (25); Anal. calc. for $\mathrm{C}_{8} \mathrm{H}_{5} \mathrm{D}_{3} \mathrm{O}_{3}: \mathrm{C} 61.93 ; \mathrm{H}+(\mathrm{D} / 2) 5.19$; found: C 61.93; H 5.16.

$\underline{4}^{\prime}-O$-methyl- $\mathrm{d}_{3}$-norbelladine: to a solution of 3-hydroxy-4-methoxy- $\mathrm{d}_{3}$-benzaldehyde (979 mg, $6.31 \mathrm{mmol}$ ) and tyramine $(866 \mathrm{mg}, 6.31 \mathrm{mmol})$ in methanol $(35 \mathrm{~mL})$ was added $4 \AA$ molecular sieves and the suspension was stirred overnight at room temperature under inert atmosphere. The mixture was filtered, cooled $\left(0^{\circ} \mathrm{C}\right)$, then $\mathrm{NaBH}_{4}$ (466 mg, $12.30 \mathrm{mmol}$ ) was added portionwise with stirring. The white suspension was stirred at room temp. for $3 \mathrm{~h}$. The solvent was evaporated and to the residue were added succesively water $(20 \mathrm{~mL})$, brine $(30$ $\mathrm{mL}$ ) and $3 \mathrm{M} \mathrm{HCl}$ until $\mathrm{pH}=8$. The suspension was extracted with EtOAc $(3 \times 50 \mathrm{~mL})$. A dark insoluble material appeared, which was kept with the organic phases and the resulting suspension was extracted with $3 \mathrm{M}$ $\mathrm{HCl}(2 \times 20 \mathrm{~mL})$ and water $(1 \times 20 \mathrm{~mL})$. The aqueous phases were gathered, basified with $5 \% \mathrm{Na}_{2} \mathrm{CO}_{3}$ until $\mathrm{pH}=8$ and the resulting suspension was extracted with EtOAc $(4 \times 50 \mathrm{~mL})$. The solution was dried $\left(\mathrm{Na}_{2} \mathrm{SO}_{4}\right)$ and the solvent evaporated. The residue was dissolved in hot ethanol $(150 \mathrm{~mL})$, the solution was filtered and, after cooling, concentrated $\mathrm{HCl}(6 \mathrm{~mL})$ was added. The solution was concentrated to dryness under vacuum and ethanol was added to the residue $(10 \mathrm{~mL})$. The suspension was kept overnight at $4{ }^{\circ} \mathrm{C}$, then filtered and the solid was washed with cooled ethanol and dried to give $656 \mathrm{mg}(2.10 \mathrm{mmol}, 33 \%$ yield $)$ of white crystals.

M.p. $208^{\circ} \mathrm{C}$ (lit.: $195^{\circ} \mathrm{C}$ for the undeuterated), (Szewczyk et al., 1988) IR (KBr): $\nu_{\max } 3454,3265,2967,2799$, $1614,1596,1515,1435,1280,1260,1223,1134,996$, $832 \mathrm{~cm}^{-1} ;{ }^{1} \mathrm{H}$ NMR (DMSO-d $): \delta 2.90\left(\mathrm{~m}, 2 \mathrm{H}, \mathrm{CH}_{2}\right.$ ), $2.99\left(\mathrm{~m}, 2 \mathrm{H}, \mathrm{CH}_{2}\right), 4.01\left(\mathrm{~s}, 2 \mathrm{H}, \mathrm{CH}_{2}\right), 6.75$ (d, $J=8.3$ $\left.\mathrm{Hz}, 2 \mathrm{H}, \mathrm{H}_{\text {arom }}\right), 6.92-7.10\left(\mathrm{~m}, 5 \mathrm{H}, \mathrm{H}_{\text {arom }}\right), 9.22$ (s, 1 $\mathrm{H}, \mathrm{OH}), 9.30$ (br s, $\left.2 \mathrm{H}, \mathrm{NH}_{2}^{+}\right), 9.43$ (s, $\left.1 \mathrm{H}, \mathrm{OH}\right) ;{ }^{13} \mathrm{C}$ NMR (DMSO-d $): \delta 30.6,47.6,49.6,112.0,115.4,117.3$, $121.2,124.2,127.2,129.5,146.4,148.2,156.2$; ESI-MS (pos. mode): $\mathrm{m} / \mathrm{z} 140[\mathrm{M}-\text { tyramine }]^{+}$(100), $277[\mathrm{M}$ $+\mathrm{H}^{+}$(65); Anal. calc. for $\mathrm{C}_{16} \mathrm{H}_{17} \mathrm{D}_{3} \mathrm{NO}_{3} \mathrm{Cl}$ : C 58.08; $\mathrm{H}+(\mathrm{D} / 2)$ 6.10; N 4.23; found: C 58.30; H 6.28; N 4.37.

Alkaloid extraction. Plant material was lyophilized, powdered and $150 \mathrm{mg}$ of powder was macerated in methanol $(10 \mathrm{~mL})$ for $24 \mathrm{~h}$, with sonication for $90 \mathrm{~min}$ in an ultrasonic bath (Transsonic 460/H Elma) at room temp. After centrifugation at $4000 \mathrm{rpm}$ for $20 \mathrm{~min}$, the mixture was filtered through $0.2 \mu \mathrm{m}$ filters, and the total methanol extract was analyzed using LC-MS and GCMS.

LC-MS analysis. The LC consisted of a U3000Dionex system, an injector with a $1 \mu$ loop and a UV detector at $280 \mathrm{~nm}$. The analytical column used was an Acclain PepMap C18 ID $1 \mathrm{~mm}$ column $(150 \mathrm{~mm} \times 3$ $\mu \mathrm{m} \times 100 \mu \mathrm{m})$ and was eluted at a flow rate of $40 \mu \mathrm{l} / \mathrm{min}$ using a gradient ranging from $0 \%$ solvent B to $100 \%$ solvent B in a time span of $36 \mathrm{~min}$. Solvent A consisted of $97.5 \% 10 \mathrm{mM}$ ammoniumbicarbonate $\mathrm{pH} 7.8$ with $2.5 \%$ methanol and solvent B consisted of $97.5 \%$ methanol and $2.5 \% 10 \mathrm{mM}$ ammoniumbicarbonate $\mathrm{pH} 7.8$. The ESi-HRMS was a micrOTOF ${ }_{Q}{ }^{\text {TM }}$ (Bruker Daltonics) apparatus.

GC-MS identification. Analyses were performed using QP2010 Shimadzu equipment operating in the EI mode at $70 \mathrm{eV}$. An AT-1 column $(25 \mathrm{~m} \times 0.32 \mathrm{~mm} \times 0.30$ $\mu \mathrm{m})$ was employed with a $33 \mathrm{~min}$ temperature program of $80-280^{\circ} \mathrm{C}$ at $10^{\circ} \mathrm{C} / \mathrm{min}$ followed by a $10 \mathrm{~min}$ hold at $280^{\circ} \mathrm{C}$. The injector temperature was $280^{\circ} \mathrm{C}$, the flow rate of the carrier gas (helium) was $0.8 \mathrm{ml} / \mathrm{min}$, the split ratio was $1: 50$. Identification of the alkaloids was performed by comparing the measured data with those of authentic compounds (galanthamine, lycorine) or with literature data as specified in the text.

\section{RESULTS AND DISCUSSION}

\section{Synthesis of the deuterated precursor of galanthamine biosynthesis}

The synthesis of $4^{\prime}$-O-methyl- $\mathrm{d}_{3}$-norbelladine was achieved in two steps, as depicted in Fig. 2. First, 3-hydroxy-4-methoxy- $\mathrm{d}_{3}$-benzaldehyde (isovanillin- $\mathrm{d}_{3}$ ) was obtained from 3,4-dihydroxybenzaldehyde, methyl- $\mathrm{d}_{3}$ iodide and potassium carbonate using a modification of a published procedure (Markey et al., 1980). Thus, crystallization was replaced by two successive column chromatographies of the crude mixture yielding pure isovanillin$\mathrm{d}_{3}$ in $49 \%$ yield, along with $19 \%$ of the undesired isomer (vanillin- $\mathrm{d}_{3}$ ).

Then, as previously described (Szewczyk et al., 1988) to get the non-labeled molecule, reductive amination with tyramine and $\mathrm{NaBH}_{4}$ afforded the target molecule, which was isolated as a hydrochloride in $33 \%$ yield.

\section{LC-MS analysis}

Shoot cultures of Leucojum aestivum grown on $\mathrm{Mu}-$ rashige and Skoog medium containing $\alpha$-naphthalene

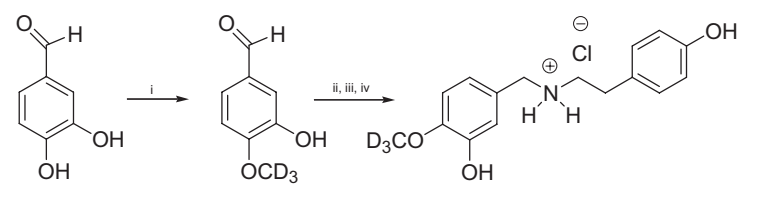

Figure 2. Steps of $\mathbf{4}^{\prime}$ - $\boldsymbol{O}$-methyl- $\mathrm{d}_{3}$-norbelladine synthesis Reagents and conditions: (i) $\mathrm{CD}_{3} \mathrm{I}, \mathrm{K}_{2} \mathrm{CO}_{3}$; (ii) tyramine, molecular sieves; (iii) $\mathrm{NaBH}_{4}$; (iv) $\mathrm{HCl}$. 
acetic acid (NAA) (10 $\mu \mathrm{M})$ and benzylaminopurine (BAP) $(5 \mu \mathrm{M})$ were subcultured in medium containing labeled precursor 4 '-O-methyl- $\mathrm{d}_{3}$-norbelladine at various concentrations $(0.05,0.10$ and $0.20 \mathrm{~g} / \mathrm{L})$ and incubated for various periods of time $(15,30$ and 40 days). Whatever the concentration of the deuterated precursor used, the growth kinetics of the shoot cultures were similar, although the growth rates (final shoot culture fresh weight - inoculum fresh weight/inoculum fresh weight) of the treated shoot cultures were lower than those of the control cultures (Table 1). These results indicated that the deuterated precursor added in the culture medium could be toxic for the shoot cultures. The lowest concentration $(0.05 \mathrm{~g} / \mathrm{L})$ of 4 '-O-methyl- $\mathrm{d}_{3}$-norbelladine led, unexpectedly, to the slowest growth of the cultures. The medium concentration $(0.10 \mathrm{~g} / \mathrm{L})$ also inhibited the growth, but the plant cultures showed the same growth rate as the control cultures after 40 days of incubation. This can be due to a correlation between shoot culture growth and the alkaloid synthesis in these tissues incubated with the labeled precursor.

After harvesting the shoot cultures, the precursor and the other alkaloids were extracted from the tissues as well as from the medium and the compounds were identified using mass spectrometry. HPLC coupled with high-
Table 1. Growth rate of Leucojum aestivum shoot culture in medium enriched with $4^{\prime}-0$-methyl- $d_{3}$-norbelladine (D3MN) at various concentrations

Data represent average of four replications with standard deviation.

\begin{tabular}{lccc}
\hline \multicolumn{5}{c}{ Growth rate } \\
\hline$[\mathrm{D} 3 \mathrm{MN}] \mathrm{g} / \mathrm{L}$ & Day 15 & Day 30 & Day 40 \\
\hline 0.00 & $0.34 \pm 0.07$ & $0.45 \pm 0.19$ & $1.03 \pm 0.15$ \\
0.05 & $0.09 \pm 0.01$ & $0.04 \pm 0.03$ & $0.30 \pm 0.09$ \\
0.10 & $0.16 \pm 0.05$ & $0.36 \pm 0.08$ & $1.05 \pm 0.07$ \\
0.20 & $0.20 \pm 0.07$ & $0.25 \pm 0.07$ & $0.83 \pm 0.09$ \\
\hline
\end{tabular}

resolution mass spectrometry (ESI/QqTOF) was used in order to identify native galanthamine and lycorine, by comparison with authentic compounds (Figs. 3a and 4a) as previously described (Ptak et al., 2009), and also deuterated galanthamine and lycorine (Figs. $3 \mathrm{~b}$ and $4 \mathrm{~b}$ ). These labeled alkaloids showed the same retention time as the native alkaloids, i.e. $33.4 \mathrm{~min}$ for galanthamine and 25.3 min for lycorine. Native galanthamine and deuterated galanthamine displayed $[\mathrm{M}+\mathrm{H}]^{+}$at a $\mathrm{m} / \mathrm{z}$ in accordance with the calculated values (288.1594 and 291.1783, respectively) (Fig. 3). Native lycorine and deuterated lycorine dis-
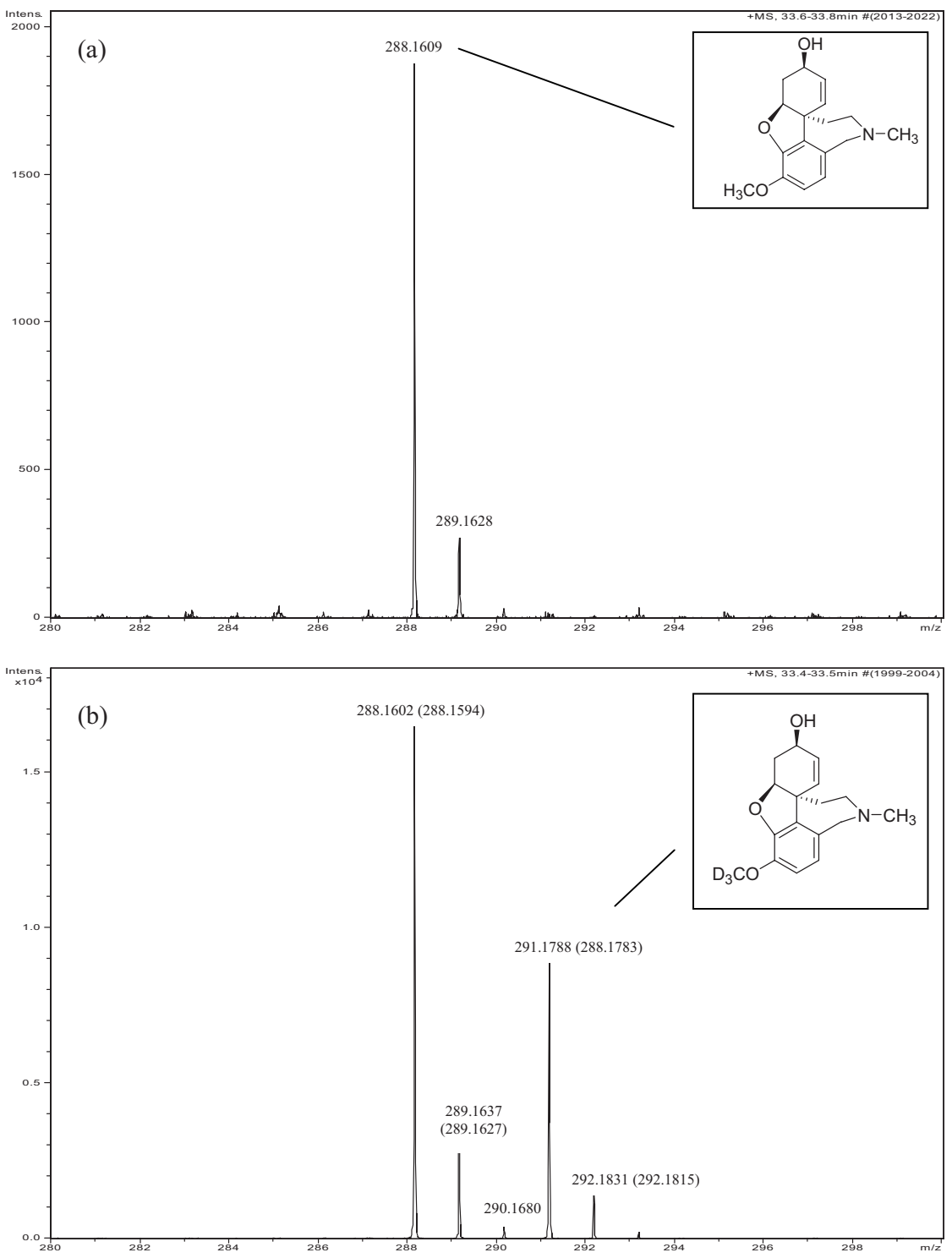

Figure 3. LC-MS mass spectra of galanthamine.

Spectra were acquired in the positiveion mode. (a) Mass spectrum of authentic galanthamine; (b) mass spectrum of native and deuterated galanthamine from an extract of shoot cultures of Leucojum aestivum. Error $<4 \mathrm{ppm}$ in both cases, theoretical masses are given in parentheses. 
Table 2. Galanthamine and lycorine identified by LC-MS in shoot cultures of Leucojum aestivum and in liquid medium Cultures were grown for 15,30 and 40 days with $4^{\prime}$ - $O$-methyl- $\mathrm{d}_{3}$-norbelladine (D3MN) at various concentrations (0.05, $\left.0.10,0.20 \mathrm{~g} / \mathrm{L}\right)$.

\begin{tabular}{|c|c|c|c|c|c|c|}
\hline & \multicolumn{3}{|l|}{ GAL } & \multicolumn{3}{|l|}{ LYC } \\
\hline Molecular mass and Formula & \multicolumn{3}{|c|}{$\mathrm{C} 17 \mathrm{H} 21 \mathrm{NO} 3$} & \multicolumn{3}{|c|}{$\mathrm{C} 16 \mathrm{H} 17 \mathrm{NO} 4$} \\
\hline$[\mathrm{M}+\mathrm{H}]+$ & \multicolumn{3}{|l|}{288.1594} & \multicolumn{3}{|l|}{288.1230} \\
\hline$[\mathrm{MD} 2+\mathrm{H}]+$ & \multicolumn{3}{|l|}{-} & \multicolumn{3}{|c|}{290.1356} \\
\hline \multirow[t]{2}{*}[\mathrm{MD}3+\mathrm{H}]{+} & \multicolumn{3}{|l|}{291.1783} & \multicolumn{3}{|l|}{-} \\
\hline & 15 Days & 30 Days & 40 Days & 15 Days & 30 Days & 40 Days \\
\hline Control shoot cultures & + & + & + & + & + & + \\
\hline Culture Medium & + & + & + & + & + & + \\
\hline Shoot cultures + $0.05 \mathrm{~g} / \mathrm{L} \mathrm{MB}^{*}$ & - &,$+ \mathrm{D} 3$ &,$+ \mathrm{D} 3$ & + & + & + \\
\hline Culture Medium & - &,$+ \mathrm{D} 3$ &,$+ \mathrm{D} 3$ & + & + & + \\
\hline Shoot cultures + $0.1 \mathrm{~g} / \mathrm{L} \mathrm{MB}^{*}$ &,+ D3 &,+ D3 &,$+ \mathrm{D} 3$ & + & + & + \\
\hline Culture Medium &,$+ \mathrm{D} 3$ &,$+ \mathrm{D} 3$ &,$+ \mathrm{D} 3$ & + & + &,$+ \mathrm{D} 2$ \\
\hline Shoot cultures + $0.2 \mathrm{~g} / \mathrm{L} \mathrm{MB}^{*}$ & + &,+ D3 & - & + & + &,$+ \mathrm{D} 2$ \\
\hline Culture Medium & + &,$+ \mathrm{D} 3$ & - & + & + &,$+ \mathrm{D} 2$ \\
\hline
\end{tabular}

GAL, galanthamine; LYC, lycorine; +, native alkaloid detected; -, no alkaloid detected; D3 or D2, deuterium-labeled alkaloid detected.
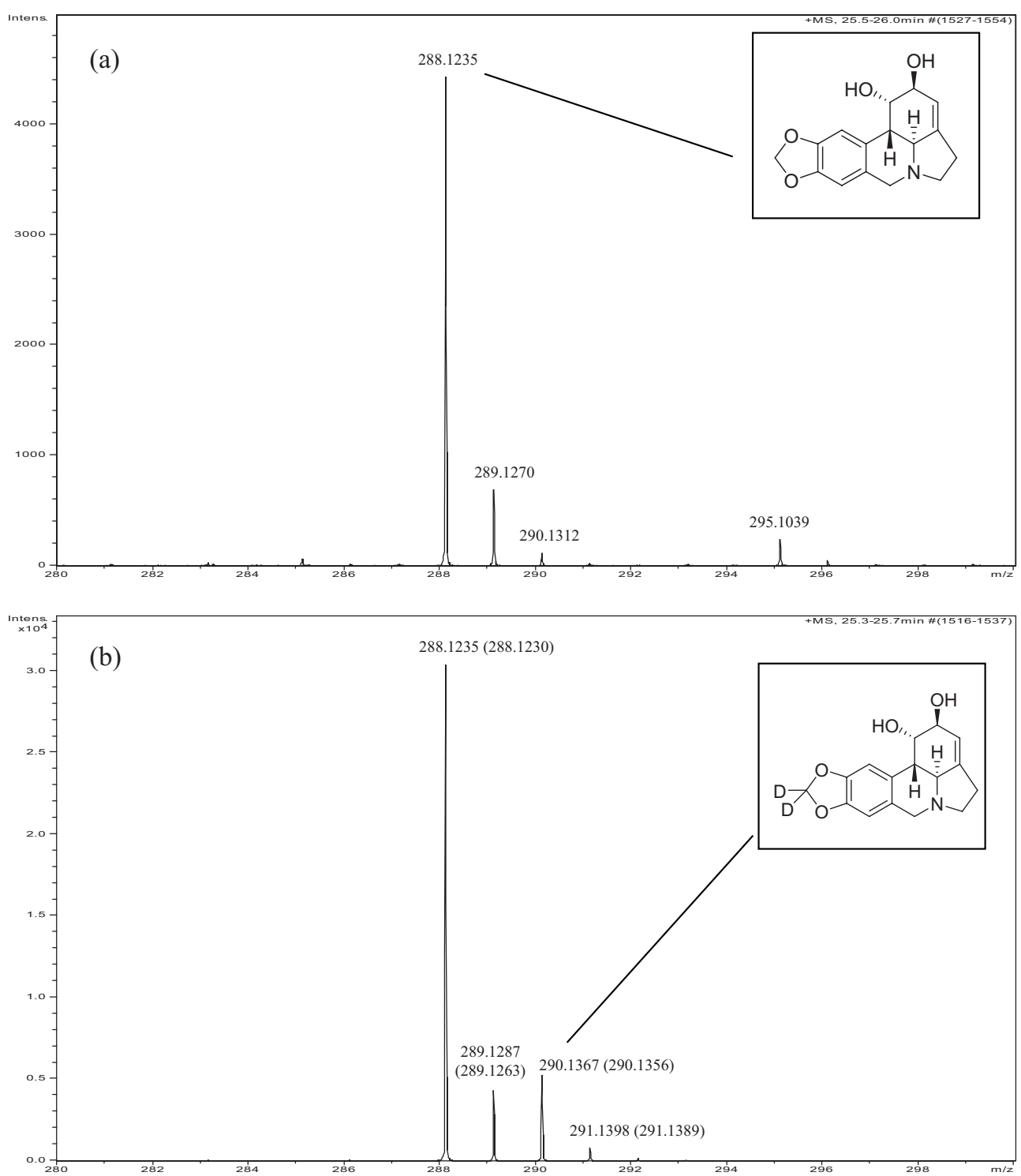

Figure 4. LC-MS mass spectra of lycorine. Spectra were acquired in the positive-ion mode. (a) Mass spectrum of authentic lycorine; (b) mass spectrum of native and deuterated lycorine from an extract of shoot cultures of Leucojum aestivum. Error < $4 \mathrm{ppm}$ in both cases, theoretical masses are given in parentheses. 


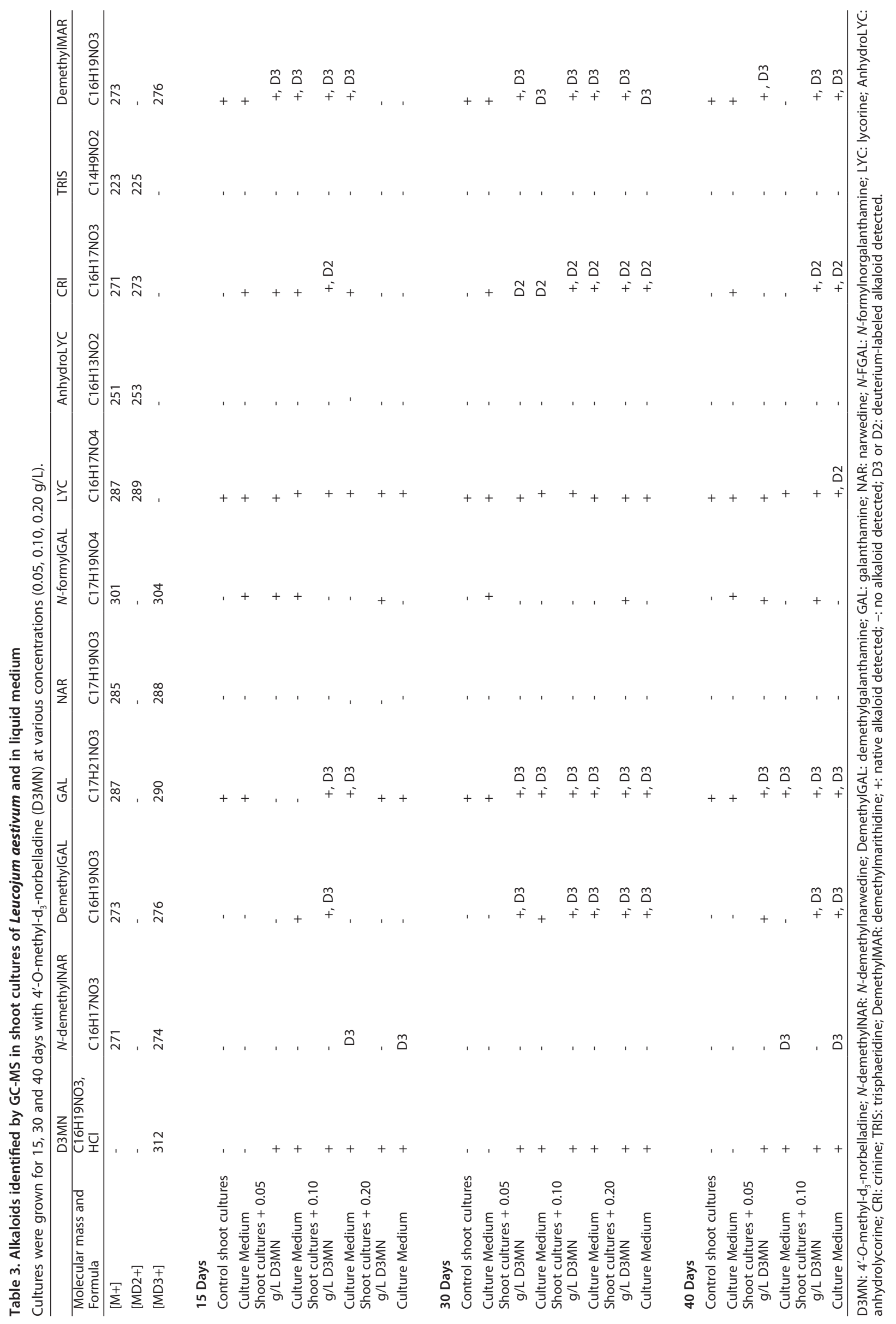


played $[\mathrm{M}+\mathrm{H}]^{+}$at a $m / z$ in accordance with the calculated values (288.1230 and 290.1356, respectively) (Fig. 4). In control shoot cultures, native galanthamine and lycorine that exhibited only the pseudo molecular ion characteristic of standard compounds were detected both in plant tissues and in liquid medium indicating that these alkaloids were able to diffuse in the culture medium. Isoquinoline alkaloids from Papaver somniferum tissue cultures were also found in liquid-medium culture (Le Flem-Bonhomme et al., 2004). The excretion of a secondary metabolite in the medium is a characteristic leading to an improved productivity in bioreactor cultures (Bourgaud et al., 2001). Regardless of the incubation duration and the concentration of precursor, the latter was detected both in shoot cultures and culture media indicating that it was transported into the plant tissue (Table 2). However, the uptake of this compound by plant tissue was not complete, as 4'-Omethyl- $\mathrm{d}_{3}$-norbelladine was still detected in culture media after 15,30 or 40 days of incubation.

Incorporation of 4 '- $O$-methyl- $\mathrm{d}_{3}$-norbelladine into both alkaloids was observed in different conditions tested. The labeled galanthamine and lycorine contained respectively 3 and 2 atoms of deuterium. Incorporation of two deuterium atoms in lycorine might result from an intramolecular cyclisation of the ortho-methoxyphenol yielding the methylenedioxy bridge. This is in accordance with what was previously proposed (Barton et al., 1963) for haemanthamine, another Amaryllidaceae alkaloid, and for benzophenanthridine (Ikezawa et al., 2007) or berberine-like alkaloids (Iwasa \& Kim, 1997; Cui et al., 2007). These results appear to constitute a new experimental proof of the proposed cyclisation mechanism.

Labeled galanthamine was observed after 15 days of incubation with $0.10 \mathrm{~g} / \mathrm{L}$ of the precursor up to 40 days of incubation. Nevertheless, at this time $0.20 \mathrm{~g} / \mathrm{L}$ concentration of precursor did not lead to galanthamine identification. On the other hand, labeled lycorine was observed only after 40 days of incubation with $0.20 \mathrm{~g} / \mathrm{L}$ of this precursor. These interesting results demonstrate that the biosynthetic pathways of these two alkaloids are in competition, 4'-O-methylnorbelladine being the common precursor. Nevertheless, a domination of the parapara' oxidative coupling on the norbelladine, leading to marithidine and crinine derivatives, has been observed in various populations of Galanthus elwesii (Berkov et al., 2004).

As for the native alkaloids, also deuterated galanthamine and lycorine diffused in the liquid culture medium.

\section{GC-MS analysis}

Capillary GC-MS was used in order to identify the various alkaloids, labeled or not, present in the complex fractions of $L$. aestivum shoot cultures. Derivatization was not required, since the Amaryllidaceae alkaloids retain their characteristic EI/MS fragmentation patterns when employing GC conditions, as reported by Kreh et al. (1995) and Tram et al. (2002). As the mass spectrometer coupled with GC works in low-resolution mode (see experimental part), some alkaloids show the same molecular mass, particularly galanthamine and lycorine $\left[\mathrm{M}^{+}\right.$ = 287], demethylnarwedine and crinine $\left[\mathrm{M}^{+}=271\right]$, and demethylgalanthamine and demethylmaritidine $\left[\mathrm{M}^{+}=\right.$ 273]. However, their fragmentation patterns and their retention times are different. The same GC-MS protocol as previously reported for $L$. aestivum alkaloids was used (Ptak et al., 2009). Seven compounds showed MS frag-



Figure 5. GC-MS chromatogram (total ion current) of the alkaloid fractions from shoot cultures of Leucojum aestivum.

Cultures were incubated 30 days with $0.10 \mathrm{~g} / \mathrm{L}$ of $4^{\prime}-O$-methyl- $\mathrm{d}_{3}-$ norbelladine. Numbered peaks correspond to the following compounds: 1: galanthamine, 2: demethylmarithidine, 3: 4'-O-methyl$\mathrm{d}_{3}$-norbelladine, 4: crinine, 5 : lycorine.

mentation patterns characteristic of the Amaryllidaceae alkaloids (Table 3) (Fig. 5). The identification of these alkaloids was performed by comparing the measured data with previously published results (Ptak et al., 2009) and with literature data (Berkov et al., 2005). Similar retention times and similar fragmentation pattern permitted us to detect unambiguously deuterated alkaloids among native ones. This way, six labeled alkaloids were identified: $d_{3}-$ demethylnarwedine, $\mathrm{d}_{3}$-demethylgalanthamine, $\mathrm{d}_{3}$-galanthamine, $\mathrm{d}_{2}$-lycorine, $\mathrm{d}_{2}$-crinine and $\mathrm{d}_{3}$-demethylmarithidine (Fig. 5, Table 3). As was shown for lycorine, two deuterium atoms were incorporated in the crinine structure, bearing the same methylenedioxy bridge. These results show that 4 '- $O$-methyl- $\mathrm{d}_{3}$-norbelladine was incorporated into the three different groups of Amaryllidaceae alkaloids that are biosynthesized by three modes of phenol coupling on the common precursor (Fig. 1). All the labeled alkaloids were observed after 15 days of incubation with the labeled precursor except $\mathrm{d}_{2}$-lycorine that was detected only after 40 days of incubation as shown above by the LC-MS analysis. Concerning the alkaloids biosynthesized by the para-ortho' oxidative coupling of 4'-O-methyl-norbelladine, labeled demethylnarwedine, demethylgalanthamine and galanthamine were detected while no labeled $N$-formylgalanthamine was observed. However, this alkaloid was detected in the extracts as a native compound. This could be due to a too short incubation time with the labeled precursor. Surprisingly, $\mathrm{d}_{3}$-demethylnarwedine was detected only in the liquid culture medium while $\mathrm{d}_{3}$-demethylgalanthamine was detected, initially, in the plant tissue only. All these results could be explained by the compartmentation in connection with enzymes that could be part of multienzyme complexes (Verpoorte et al., 1999). It has been reported that compartmentation plays a major role in the regulation of secondary metabolite pathways. For example, the biosynthesis of terpenoid indole alkaloids requires at least three compartments, the plastids, the cytosol and the vacuole (Verpoorte et al., 1997). The absence of native or labeled narwedine was noted. Concerning the alkaloids biosynthesized by the para-para' oxidative coupling, labeled crinine and demethylmarithidine were detected. Both alkaloids were first observed after 15 days of incubation with the labeled precursor respectively at 0.10 and $0.05 \mathrm{~g} / \mathrm{L}$. It is noteworthy that these labeled compounds appeared simultaneously with $\mathrm{d}_{3}$-galanthamine suggesting that the competition between the three modes of phenol coupling on the common precursor O-methyl-norbella- 
dine was in favour of the para-ortho' (galanthamine synthesis) and the para-para' (crinine and demethylmarithidine synthesis) oxidative couplings. No native nor labeled trisphaeridine was detected in the extracts. Further investigations with the labeled precursor are still required to better understand Amaryllidaceae alkaloid metabolism in tissue cultures of $L$. aestivum, in particular the flux rates between the biosynthetic intermediates. In conclusion, the common precursor $4^{\prime}-\mathrm{O}$-methyl- $\mathrm{d}_{3}$-norbelladine is biotransformed in shoot cultures of $L$. aestivum and gives labeled alkaloids previously reported in the literature but using ${ }^{13} \mathrm{C}$ or ${ }^{14} \mathrm{C}$-labeled precursor.

\section{Acknowledgements}

The authors thankfully acknowledge Brigitte Fernette for NMR recording, Sandrine Adach for elementary analyses, and Jean-Pierre Joly for judicious suggestions.

\section{REFERENCES}

Barton DHR, Cohen T (1957) In Festschrift Arthur Stoll; p 117. Birkhäuser, Basel.

Barton DHR, Kirby GW, Taylor JB, Thomas GM (1963) Phenol oxidation and biosynthesis. Part VI. The biogenesis of Amaryllidaceae alkaloids. I Chem Soc 4545-4558.

Bastida J, Lavilla R, Viladomat F (2006) Chemical and biological aspects of Narcissus alkaloids. In The Alkaloids. Cordell GA, eds, 3: 87-179. Elsevier Inc.

Berkov S, Sidjimova B, Evstatieva L, Popov S (2004) Intraspecific variability in the alkaloid metabolism of Galanthus elwesii. Phytochemistry 65: 579-586.

Berkov S, Pavlov A, Ilieva M, Burrus M, Popov S, Stanilova M (2005) CGC-MS of alkaloids in Leucojum aestivum plants and their in vitro cultures. Phytochem Anal 16: 98-103.

Bernfeld P (1963) In Biogenesis of Natural Compounds; p 985. Pergamon Press Oxford,

Bourgaud F, Gravot A, Milesi S, Gontier E (2001) Production of plant secondary metabolites: a historical perspective. Plant Sci 161: 839_ 851.

Cui W, Iwasa K, Sugiura M, Takeuchi A, Tode C, Nishiyama Y, Moriyasu M, Tokuda H, Takeda K (2007) Biotransformation of phenolic 1-benzyl- $N$-methyltetrahydroisoquinolines in plant cell cultures followed by LC/NMR, LC/MS, and LC/CD. J Nat Prod 70: 17711778 .

Eichhorn J, Takada T, Kita Y, Zenk MH (1998) Biosynthesis of the Amaryllidaceae alkaloid galanthamine. Phytochemistry 49: 1037-1047.
Guillou C, Beunard J, Gras E, Thal C (2001) An efficient total synthesis of $( \pm)$-galanthamine. Angew Chem Int Ed 40: 4745-4746.

Herbert RB (2001) The biosynthesis of plant alkaloids and nitrogenous microbial metabolites. Nat Prod Rep 18: 50-65.

Ikezawa N, Iwasa K, Sato F (2007) Molecular cloning and characterization of methylenedioxy bridge-forming enzymes involved in stylopine biosynthesis in Escholzia californica. FEBS J 274: 1019-1035.

Iwasa K, Kim CW (1997) Biotransformations of protoberberines in cell cultures of Dicentra spectablis. Phytochemistry 46: 1359-1363.

Kornienko A, Evidente A (2008) Chemistry, biology, and medicinal potential of narciclasine and its congeners. Chem Rev 108: 1982-2014.

Kreh M, Matusch R, Witte L (1995) Capillary gas chromatographymass spectrometry of Amaryllidaceae alkaloids. Phytochemistry 38: 773-776.

Kukhanova M, Victorova L, Krayevsky A (1983) Peptidyltransferase center of ribosomes: on the mechanism of action of alkaloid lycorine. FEBS Lett 160: 129-133.

Le Flem-Bonhomme V, Laurain-Mattar D, Fliniaux MA (2004) Hairy root induction of Papaver somniferum var. album, a difficult-to-transform plant, by A. rhizogenes LBA 9402. Planta 218: 890-893.

Marco-Contelles J, Leon R, de Los Rios C, Guglietta A, Terencio J, Lopez MG, Garcia AG, Villarroya M (2006) Novel multipotent tacrine-dihydropyridine hybrids with improved acetylcholinesterase inhibitory and neuroprotective activities as potential drugs for the treatment of Alzheimer's disease. I Med Chem 49: 7607-7610.

Markey SP, Powers K, Dubinsky D, Kopin IJ (1980) General method for the synthesis of methyl isotope labeled cathecolamine metabolites. Preparation of 4-hydroxy-3-methoxy-d3-(madelic acid, phenylacetic acid and phenylethylene glycol). J Labelled Comp Radiopharm 17: 103-114.

Murashige T, Skoog F (1962) A revised medium for rapid growth and bioassays with tobacco cultures. Physiol Plant 15: 473-497.

Ptak A, El Tahchy A, Dupire F, Boisbrun M, Henry M, Chapleur Y, Moś M, Laurain-Mattar D (2009) LCMS and GCMS for the screening of alkaloids in natural and in vitro extracts of Leucojum aestivum. J Nat Prod 72: 142-147.

Szewczyk J, Lewin AH, Carroll FI (1988) An improved synthesis of galanthamine. J Heterocyclic Chem 25: 1809-1811.

Szlávik L, Gyuris A, Minarovits J, Forgo P, Molnar J, Hohmann J (2004) Alkaloids from Leucojum vernum and antiretroviral activity of Amaryllidaceae alkaloids. Planta Med 70: 871-873.

Tram N, Mitova M, Bankova V, Handjieva N, Popov S (2002) GS-MS of Crinum latifolium L. alkaloids. Z Naturforsch 57: 239-242.

Verpoorte R, van der Heijden R, Moreno PRH (1997) Biosynthesis of terpenoid indole alkaloids in Catharanthus roseus cells. In The Alkaloids. Cordell GA, eds, 49: 221-299. Academic Press, San Diego.

Verpoorte R, van der Heijden R, ten Hoopen HJG, Memelink J (1999) Metabolic engineering of plant secondary metabolite pathways for the production of fine chemicals. Biotechnol Lett 21: 467-479. 